\title{
La tétralogie et la trilogie attique
}

Marie Delcourt

\section{Citer ce document / Cite this document :}

Delcourt Marie. La tétralogie et la trilogie attique. In: L'antiquité classique, Tome 7, fasc. 1, 1938. pp. 31-50;

http://www.persee.fr/doc/antiq_0770-2817_1938_num_7_1_3066

Document généré le 24/01/2017 


\title{
LA TÉTRALOGIE E'I LA TRILOGIE AT'TIQUE
}

\author{
par Marie Deicount.
}

Les biologistes nous ont appris à reconnaître, dans le corps des êtres vivants, des organes rudimentaires qui ont probablement servi autrefois mais qui, correspondant à un fonction aujourd'hui disparue, se sont atrophiés. De même, lorsqu'un poète modifie un mythe, il lui arrive de garder un détail qui provient dela légende antérieure; ce détail a vivement frappé son imagination et il ne s'est pas aperçu que, dans le tissu nouveau auquel il l'incorpore, il est, ou inutile, ou disparate, ou même contradictoire. Zielinski, empruntant la terminologie de Wundt, voit dans la mythopée antérieure une représentation induisante, dans le détail rudimentaire une représentation induite.

Exemples: 1. Dans la légende ancienne, Néoptolème est tué à Delphes sur l'ordre d'Apollon ou des prêtres du temple. Euripide, dans Andromaque ( $\left.{ }^{1}\right)$, imagine une tout autre version où le meurtrier est Oreste. Le meurtre peut, dès lors, se placer en n'importe quel endroit où les deux rivaux se rencontrent. Mais le détail de la mort à Delphes continue à s'imposer à l'esprit du poète, lequel envoie Oreste en Phocide pour tendre une embuscade à Néoptolème. Ce détail n'est nullement contradictoire avec le reste de la pièce, mais il est gênant, parce qu'on est obligé d'admettre qu'entre le vers 1008 et le vers 1070 Oreste a eu le temps d'aller de Buthrote à Delphes, et le messager le temps de revenir de Delphes à Buthrote. Le beau récit qui suit, si ample, si juste, est néanmoins la mise en ouvre d'un détail rudimentaire, devenu inutile dans cette version de la légende. Pausanias a encore vu à Delphes, où l'on s'en tient à la mythopée traditionnelle, le tombeau de Néoptolème. Mais Vir-

(1) Mais non dans Oreste (1657), où il revient à la mythopée traditionnellè, qui était encore celle de l'Hermione de Sophocle. 
gile, qui met l'accent sur le fait qu'Oreste est le meurtrier, ne sait plus que faire de l'épisode delphique et montre Néoptolème assassiné au pied de ses autels nationaux $\left(^{1}\right)$.

2. L'exposition d'Oedipe se racontait de plusieurs façons. Ou bien il avait été abandonné dans la montagne, pendu par les pieds, ou bien il avait été remis par un berger à un autre (ou encore il avait été déposé dans un berceau flottant, comme Moïse et Romulus). l)ans le premier cas, on pouvait l'identifier grâce à la cicatrice, dans le second, en confrontant les deux bergers. Sophocle, dans Oedipe-Roi, suit la seconde légende, mais le détail des pieds percés s'impose à lui et il le garde malgré la disparate, car on ne voit pas à quel moment le nouveau-né, remis par ses parents au berger thébain et par celui-ci au berger corinthien, aurait pu ètre pendu en plein air. La cicatrice au pied, dans Oedipe-Roi, est un détail rudimentaire $\left({ }^{2}\right)$.

3. Dans les Choéphores (560), Oreste se propose de rester devant le palais jusqu'à ce qu'un passant s'étonne de voir ainsi l'hospitalité violée et le fasse entrer. Alors il tuerait Egisthe sur son trône. I es choses se passent tout autrement dans la pièce .Le plan d'Oreste apparaît donc comme un détail rudimentaire qui révèle ce qui se passait dans une forme plus ancienne de la légende, probablement, ici, chez Stésichore, lequel présentait la vengeance du fils comme Homère l'avait fait, e'est à dire comme le retour de l'héritier légitime détrônant l'usurpateur $\left(^{3}\right)$.

Thadée Zielinski a essayé de faire une étude systématique des détails rudimentaires et de reconstituer grâce à eux des mythopées perdues $\left({ }^{4}\right)$. Cette méthode est délicate à employer, car un détail sans objet dans une pièce peut ètre tout autre chose qu'un souvenir d'une cuvre plus ancienne. Au lieu d'être une allusion involontaire à un détail mort qui n'a pas cessé d'habiter la mé-

(1) P'aus., x, 24, 5. - Enéide, III, 332. - Cif. Marie Delcourt, La légende de la mort de Néoptolème, Revue belge de ph. et d'hist., 1923, p. 685.

(2) Carl Robert, Gedipus, p. 289, a signalé la contradiction entre le vers 1026, où le berger corinthien raconte avoir trouvé l'enfant, et les vers 1038-40, où il dit l'avoir reçu d'un autre. Ce dernier détail seul sert à l'action.

(3) Cif. Choéph. éd. Mazon, n. des vers 562 et 573.

(4) Th.Zielinski. Tragodumenon libri $1 I I$, Cracovie, 1925, l.I. de locis tragoediae Graecae rudimentalibus, -.. du même, Pour reconstituer les tragédies perdues, dans la Rov. belge de phil. et d'hist.,1927, pp. 593 sqq et 1928 pp. 5 sqq. 
moire, ce pourraît être une allusion volontaire à un épisode auquel l'auteur se propose de donner une importance nouvelle. L'intérêt que porte le chœur d'Agamemnon au sort de Ménélas s'explique, nous le savons, par le drame satyrique perdu. Mais, si nous ignorions que la tétralogie se terminait par un Protée, nous serions bien lentés de voir, dans le dialogue des vers $61 \bar{j}$ et suiv., un détail rudimentaire que nous expliquerions comme survivant d'une légende où c'est Ménélas qui venge Agamemnon, comme il se propose de le faire dans l'Odyssée (IV, 546). D'autre part, un détail sans influence sur le reste de l'action peut avoir une importance purement psychologique. Ainsi, dans la pièce de Sophocle, Egisthe se propose d'enfermer Electre dans un cachot si elle continue à l'importuner; et Electre, au moment où elle croit Oreste mort, se décide à tuer elle-même Egisthe. Plus tard, il n'est plus question d'aucun de ces deux projets. Si nous connaissions moins bien la légende, nous pourrions croire qu'il existait une version où le meurtre était commis par Electre. Les deux détails servent uniquement à mettre en valeur le caractère indomptable d'Électre qu'aucune menace ne peut briser. Wilamowitz, dans son article célèbre sur les Deux Electre ( $\left.{ }^{1}\right)$, dit qu'ici Sophocle s'est copié lui-même et qu'il a introduit dans Electre, comme un accessoire inutile, le même caveau qui joue un rôle efficace dans Antigone. L'explication est un peu grosse, mais elle met en lumière une chose qu'il ne faut pas oublier, à savoir qu'un détail peut rester rudimentaire sans pour cela avoir appartenu à une mythopée antérieure, mais simplement parce qu'il était subordonné à un dessein qui n'était pas le progrès de l'action. Cependant, l'interprétation des détails rudimentaires a permis à Zielinski des reconstitutions intéressantes, sinon toujours convaincantes. Elle paraît surtout féconde lorsqu'on en limite l'emploi à des ensembles restreints.

Dans son livre Die Trilogie des Aischylos, sous-intitulé Formgesetze und Wege der Rekonstruktion $\left({ }^{2}\right)$, M. Frans Stoessl essaie d'étendre la méthode à un problème nouveau. Zielinski veut, par elle, atteindre la mythopée antérieure; Stoessl lui demande de le renseigner sur la marche même des pièces perdues. Il combine donc le problème du contenu et le problème de la structure. Je ne

(1) Die beiden Elektren, Hermes, 1883, p. 217.

(2) Baden bei Wien, 1937, 264 pp. 
crois pas que, depuis les temps lointains de Hartung et d'Euripides restitutus, on ait essayé de suivre scène à scène la marche d'une tragédie perdue. Hartung le faisait en partant des fragments; il travaillait sur un auteur que, grâce aux papyrus, nous connaissons mieux que lui; les textes retrouvés ont rarement confirmé ses reconstructions $\left(^{1}\right)$. M. Stoessl part d'une idée de la tragédie et il ne s'occupe d'intégrer les fragments qu'après avoir reconstruit un schème qui lui paraisse satisfaisant. Et, le principe de ce schème, il le demande aux lois qui régissent la composition trilogique.

C'est pour découvrir cette loi qu'il fait appel à la méthode des rudiments. De même qu'il existe, dit-il, des détails rudimentaires qui nous renseignent sur la mythopée antérieure, il y a aussi des scènes rudimentaires, qui ne servent à rien dans l'œuvre où elles figurent. Il faut chercher la raison de leur existence ailleurs, dans l'ensemble trilogique dont elles font partie. Là, elles s'expliquent: elles servent de contre-poids à une scène parallèle, mais utile dans une autre tragédie. Cherchons donc à détecter la présence d'une scène inutile; elle nous avertit que, dans une tragédie symétrique à la nôtre, il y avait, à la même place, une scène indispensable à laquelle l'auteur s'est senti obligé de donner un pendant.

Tous les auteurs qui ont étudié les tragédies grecques ont essayé de voir comment et pourquoi les poètes les groupaient en ensembles ; le problème se pose pour Euripide (quoi qu'il ne compose plus guère de trilogies liées) comme pour Eschyle, mais en d'autres termes. 'Tous ceux qui s'y attachent se heurtent une difficulté maîtresse, qui est que nous ne possèdons qu'une seule trilogie complète; encore est-elle privée de son drame satyrique et le prologue des Choéphores est-il mutilé. De plus, aucun théoricien ancien ne paraît s'être intéressé au problème du lien trilogique ou tétralogique ni en avoir mème senti l'existence. A cette difficulté primordiale, qui est la paucité des documents, M. Stoessl en ajoute une autre, qui est d'introduire dans ses prémisses la notion de scène inulile. A vrai dire,il prétend ici s'appuyer au contraire sur un terrain solide: comme presque tous les critiques allemands, il donne force de loi au passage fameux de la Poétique où, à trois ou quatre reprises,

(1) Rien de plus curieux que de comparer l'image qu'il se fait d'Ilypsipyle avec ce que nous en savons depuis que de longs fragments ont été retrouvés. On trouvera un résumé de la question dans Euripidis Hypsipyla, ed. G. Italie, diss. de Leyde, 1923. 
Aristote affirme la primauté de l'action sur les caractères $\left({ }^{1}\right)$. Mais précisément l'insistance d'Aristote est suspecte; le ton du passage indique une polémique. Au surplus, si la primauté de l'action avait vraiment été une règle universellement admise par les poètes du ve siècle, on s'expliquerait mal l'existence de tant de tragédies sans action $\left({ }^{2}\right)$. En tout cas, il est imprudent, lorsqu'on veut saisir un fait général duquel on va déduire quelque chose, de partir d'une esthétique postérieure d'un siècle à l'apparition de ce fait.

Voyons maintenant, dans le concret, comment M. Stoessl raisonne.

Agamemnon et les Choéphores sont deux pièces symétriques. Les introductions sont parallèles et formées de trois scènes :

Le veilleur. - Chour et Clytemnestre. - Le héraut (1-680). Oreste. - Electre et choeur. - Electre avec les offrandes d'Oreste (1-305). Mais dans, les Choéphores, tout sert au progrès de l'action, tandis que, dans Agamemnon, la scène du veilleur et celle du héraut font double emploi. Tout donne à penser que c'est Eschyle qui a introduit dans la tragédie le veilleur homérique. A partir du moment où c'est le veilleur qui annonce que Troie est prise, la scène du héraut devient inutile; elle n'est plus qu'un rudiment attestant un ćtat antérieur. Eschyle l'a gardée pour faire pendant au scènes correspondantes des Choéphores.

Le parallélisme continue; le chœur des vieillards exprime une angoisse croissante ( $A g$. 681-781), le chœur des jeunes filles exprime une espérance croissante (Ch. 306-478) ; car, si les deux tragédies ont une structure semblable, elles sont gouvernées par des sentiments inverses; on va dans l'une de la joie au deuil, dans l'autre du deuil à la joie $\left({ }^{3}\right)$. La seconde phase de l'action commence dans

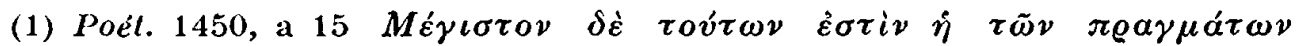

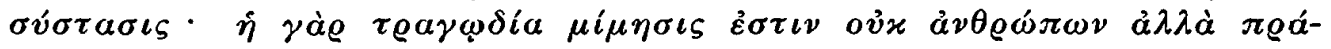

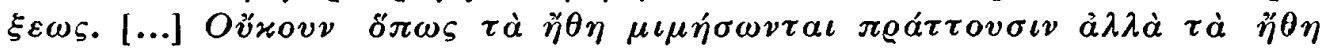

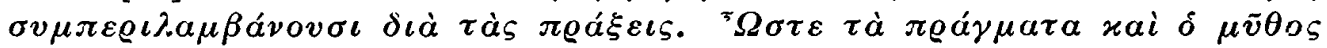

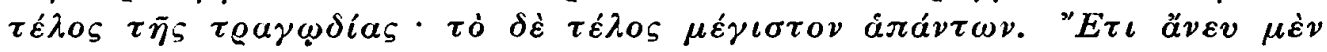

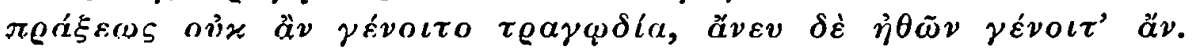

(2) "Les Troyennes sont la pièce que les critiques littéraires ont généralement choisie pour démontrer à Euripide qu'il ne savait pas construire une tragédie. Je n'éprouve nul besoin de mettre le grand poète en règle avec les exigences d'une esthétique surannée". L. Parmentier, dans son édition des Troyennes,notice, p. 2. Les censeurs dont il parle sont ceux qui prennent à la lettre le passage de la Poétique.

(3) Lesky, Die Orestie des Aischylos, Hermes, 1931, pp. 204-5, signale aussi 
Ag. en 810, avec le retour du roi, dans les $C h$. en 479 , avec la conjuration du père mort; puis, l'un et l'autre choeur (Ag. 97j-103.1, Ch. 585-651) dit son trouble, son ignorance, ici, en présence d'Oreste muet, là, en présence de Cassandre muette. La scène de Cassandre (1035-1342) correspond à celle où Oreste se dit mort à Clytemnestre (652-867). La scène de Cassandre est inutile, donc elle a été composée comme responsion au dialogue Oreste-Clytemnestre, lequel est indispensable au progrès de l'action. Les deux scènes de meurtre se correspondent ( $\mathrm{Hg}$. 1342-1371, Ch. 870-973). Comme il y a dans les Choéphores deux personnes à tuer séparément, Eschyle, pour allonger l'épisode dans Agamemnon, compose le dialogue des choreutes qui ne sert à rien, sinon à donner un contre-poids, dans la première pièce, à une scène indispensable de la seconde.

Réapparition des meurtriers (Ag. 1372-1575, Ch. 974-1062). Ils ne triomphent pas longtemps; les Argiens menacent Egisthe, les Furies menacent Oreste.

De cette étude, M. Stoessl croit pouvoir dégager les lois suivantes :

La premiere et la seconde pièce d'une trilogie se correspondent comme strophe et antistrophe. La troisième pièce, qui a une composition toute différente, sert de clausule à l'une et à l'autre comme l'épode dans un système pindarique (p. 46).

La pièce qui impose son schème à l'autre, c'est la seconde. L'économie d'Agamemnon est subordonnée à celle des Choéphores (p. 39).

Au parallélisme de la composition correspond un renversement

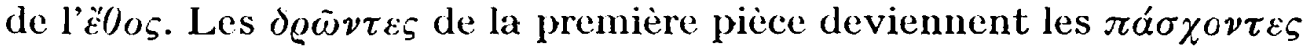
de la seconde et réciproquement. La combinaison de ces deux lois permet à M. Stoessl de découvrir des symétries qui n'avaient pas été détectées avant lui $\left({ }^{1}\right)$.

En possession de cett instrument. M. Stoessl s'efforce de retrouver, non seulement le contenu, mais la structure des trilogies perdues. L'étude des fragments n'est jamais pour lui un point de départ.

un jarallélisme entre les deux pièces. Il n'en tire du reste pas les mêmes conclusions que M. Stoessl. Au surplus, on se demande si deux pieces consacrées l'une à un crime, l'autre a la vengeance, ne seront pas nécessairement empreintes d'un certain parallélisme qui tient au sujet lui-mème et non pas à une esthétique particulière.

(1) Dans Agammenvn, les SQw̃vtes, séparés au débul de la pièce, sont rẻunis à la fin; les $\pi a ́ \sigma \chi o \nu \tau \varepsilon \varsigma$, unis au début, sont séparés à la fin. Dans les Choéphores, c'est le contraire. 
Il commence par rechercher un plan, après quoi il $\mathrm{y}$ intègre ce qu'on sait par ailleurs.

Exemple: le Desmotès est une première pièce; donc, il a été composé sur le Luomenos. Du reste, l'étude du texte le prouve surabondamment. Le Desmotès est plein de détails et de scènes rudimentaires qui s'expliquent seulement si l'on y voit le pendant d'éléments utiles dans le Luomenos (pp. 114 sqq).

Prométhée est effrayé par l'approche du chœur des Océanides qui sont cependant ses amies. Cela ne s'explique pas. Mais, dans le Luomenos, le chour était composé de Titans hostiles et, là, le "thème de la peur" était bien à sa place.

Océan est le père des jeunes filles, de la présence desquelles il ne tient du reste aucun compte, sans que l'on sache bien pourquoi. Leur parenté ne joue aucun rôle dans la pièce et la scène d'Océan elle-même ne sert à rien. Mais, dans le Luomenos, Terre était la mère des Titans ; comme elle prenait parti pour Prométhée contre eux, il était naturel qu'elle ignorât leur présence. La scène inutile Océan-Prométhée servait simplement à contre-peser une scène utile Terre-Prométhée. Le parallélisme se combine avec un renversement de l'ź$\theta 0 \varsigma$ : un Dieu hostile et ses filles favorables au héros; une Déesse favorable et ses fils hostiles au héros.

A la scène d'Io qui ne sert à rien correspondait dans le Luomenos une scène d'Héraclès. A l'errante faible, tourmentée, qui interroge Prométhée et lui demande secours, s'opposait un errant fort, victorieux, que Prométhée interrogeait et qui le libérait enfin. Ainsi, M. Stoessl trouve confirmé dans le Luomenos tel qu'il l'a reconstitué la loi de la primauté de la seconde pièce (p. 130) loi qui, à vrai dire, a dirigé toute la reconstitution - et il en conclut (p. 135) qu'Accius a dû puiser dans la pièce centrale les éléments de son adaptation.

Il est inutile de souligner le fait que cette reconstruction repose sur un raisonnement circulaire. Celle des autres trilogies est bien plus arbitraire encore. M. Stoessl cède trop souvent au désir de ceux qui veulent trop prouver et qui, après avoir entouré une hypothèse des précautions les plus honnêtes, retirent leurs réserves à la page suivante, considèrent l'hypothèse comme démontrée et la prennent comme pierre d'assise pour une nouvelle hypothèse. Après avoir reconstitué les Egyptiens (dont nous ignorons tout) d'après le plan des Suppliantes, il croit pouvoir affirmer (p. 113) que la symétrie de ces deux pièces était plus parfaite encore que celle 
d'Agamemnon et des Chó́phores, et il en conclut qu'Eschyle a dû, dans sa vicillesse, détendre la responsion rigoureuse qu'il pratiquait vingt ans auparavant. De même, il décrit Phineus d'après le patron des Perses et d'après la légende suivie par Apollonius de Rhodes (1) et il admet, dans Phineus, deux chours, ce qui l'amène à écrire : "Peut-être dans les Perses y avait-il aussi deux chours, comme dans Phineus" (p.169). Avec des inductions de ce genre, on devrait pouvoir, non seulement s'imaginer, mais récrire toutes les pièces perdues. Cependant, on lit le livre d'un bout à l'autre avec intérêt et profit. La rigueur de la thèse n'empêche pas l'auteur de bien voir les détails; il les éclaire souvent d'un jour nouveau ( $\left.{ }^{2}\right)$.

Comme on a pu le voir plus haut, la division en stasima et en épisodes ne joue aucun rôle dans les analyses de M. Stoessl et, lorsqu'il introduit une coupe, elle ne respecte pas nécessairement la distinction entre les parties récitées et les parties chantées. Voilà de quoi nous mettre en défiance. Si réellement la première pièce avait été taillée scéniquement sur le patron de la seconde, les poètes auraient naturellement marqué le parallélisme dans le cadre même du système formé par l'alternance du chant et de la déclamation. Faute de quoi, personne n'a dû le remarquer, et c'est en effet ce qui semble bien être arrivé.

La musique donnait peut-être, aux tragédies qui se suivaient, une unité dont les éléments nous échappent. Léon Parmentier était convaincu que les trois chocurs successifs utilisaicnt des timbres vocaux diffèrents. Voix de vieillards ou de jeunes gens dans 1 lexandre, d'hommes dans Palamède, de femmes dans les Troyennes; barytons dans Agamemnon, soprani dans les Choéphores, alti dans les Euménides. L'hypothèse est bien séduisante : elle nous ferait toucher, dans le tétralogie classique, quelque chose d'analogue aux mouvements d'une symphonie (et le drame satyrique correspondrait, sans du reste que nous sachions bien comment, au scherzo).

(1) Au surplus, imaginer (p. 163) qu'Apollonius (II, 178-498) suivait exactement la version développée dans le Phineus d'lischyle, c'est lui enlever toute invention personnele et sortir de toule vraisemblance. Il n'y a probablenent, dans la littérature de l'humanité, aucun exemple de deux mythopées poétiques coüncidant exactement entre elles.

(2) Ainsi , en étudiant Prométhée, il réfute minutieusement ct intelligement la thèse de Wilh. Schmidt, qui croit la pièce inauthentique : Unters. zum gefess. Prometheus, Stuttgart, 1922. Tuebinger Beiträge, IX. 
Mais elle ne va pas sans difficultés. En effet, les choreutes étaient tous des hommes et les didascalies indiquent clairement.que les poète disposait d'un seul chœur. Cependant même posé en ces termes, le problème n'est pas insoluble. L'écart entre les timbres pouvait être plus étroit que ce que nous imaginons; les choreutes étaient assez nombreux pour que le poète pût les répartir d'après les voix et ne pas les employer tous également dans chacune des quatre pièces. Contrairement à l'hypothèse de Parmentier, on admet aujourd'hui que, dans la trilogie des Suppliantes, le chœur ait été chaque fois composé des cinquante Danaïdes $\left(^{1}\right)$. Assurément, si un seul témoignage ancien nous avait conservé ce renseignement, rien ne nous autoriserait à le metter en doute; mais le peu que nous savons des ensembles trilogiques comporte précisément l'apparition de chœurs différents.

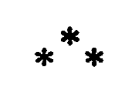

M. Stoessl, on le voit, ne tient aucun compte, dans la tragédie, des articulations que la musique et la danse rendaient immédiatement sensibles aux spectateurs. Son livre, où, sauf erreur, le nom d'Aristote n'est pas prononcé, est entièrement dominé par la notion aristotélicienne de l'action.Cette façon de voir est assez généralement celle de l'école allemande. Pour elle, la tâche du poète est avant tout la solution du problème technique posé par Aristote. Si elle a pris cette attitude, c'est en réaction contre la tendance qui s'est marquée dans la critique à la fin du siècle dernier, lorsqu'on expliquait le passage d'une forme d'art à une autre comme une étape d'une évolution naturelle, d'un développement organique, comme un simple aspect d'une sorte de transformisme mystérieux, où la volonté de l'artiste est comptée pour rien $\left({ }^{2}\right)$. Les critiques alle-

(1) Depuis A. Nathansky, Des Aischylos Danais, Wiener Studien, 32, 1910, p. 7 (dans la première pièce, les chorentes représentant les Danaïdes et leurs suivantes ; dans la seconde, les Danaïdes et les Égyptiades; dans la troisième, les Danaïdes et les juges); suivi par Croiset, Eschyle, pp. 65 sqq. et par Stoessl, op. cit., p. 107.

(2) Tout a fait caractéristique de cette façon de voir est l'article de Croiset, Les origines de la tétralogie grecque, Rev. des ét. gr. 1888, p. 369 sqq. Roy Flickinger, dans The greek theatre and its drama, p. X. signale que le point de vue technique a généralement échappé aux critiques avant 1910 ; il excepte seule- 
mands d'aujourd'hui mettent fortement l'accent sur l'apport personnel de chaque poète, et, dans le travail de ceux-ci, sur ce qui est solution consciente d'un problème technique. Il y a là, assurément, une réaction utile. Encore serait-il sage de ne pas la pousser trop loin.

C'est ainsi qu'il y a toute une littérature sur la composition de l'Electre de Sophocle, simplement parce que l'héroïne n'agit, ni directement, ni par influence, sur Oreste. Martha Koopmann (1) propose une solution en suggérant qu'Electre, prêtresse du mort Agamemnon, amène celui-ci, par la force de ses prières, à entrer dans le jeu. On trouvera dans cette dissertation (p.6, n.) la bibliographie de la question. Pour saisir la différence de ce point de vue avec celui de la critique française, il faut relire, dans l'article de Léon Parmentier sur Une scène de l'Electre de Sophocle $\left({ }^{2}\right)$, les deux pages consacrées à développer cette idée: "C'est dans l'âme de l'héroüne que se passe tout le drame, c'est dans ses sentiments que réside l'intérêt tout entier. Beaucoup de scènes ne servent pas directement à l'action, mais toutes ont pour objet de mettre en relief le caractère d'Electre ". Nous voilà bien loin de la primauté arsitotélicienne de l'action sur la psychologie. On peut se demander si la formule "für die Handlung wirkungslos", qui revient à chaque instant sous la plume des critiques allemands, aurait eu un sens quelconque pour des spectateurs grees du $v^{e}$ siècle.

Au surplus, même en prenant les choses du point de vue de l'action et de la vraisemblance, on jugera souvent la technique

ment les recherches de Adolf Gross : die Stichomythie in der gr. Trag. u. Kom. (1905) et de F. Leo : der Monolog im Drama (1908). — La nouvelle méthode allemande règne dans le livre posthume de Tycho de Wilamowitz, Die Dramatische Technik des Sophocles, Phil. Unters. 1917, XXII' cahier. L'auteur s'efforce de démontrer, non seulement que les caractères sont subordonnés à l'action, mais encore que l'action se réduit a ce que voil le spectateur. Sophocle ne s'occupe pas de ce qui se passe derrière la scène et escamote des évinements dont la suite échappe dès qu'on essaie de se l'imaginer de plus près. "Il se comporte avec ses personnages bien plus souverainement que ne pourrait le faire un dramaturge moderne. Il les fait apparaître et disparaitre comme il lui plaît et même ce qu'ils font sur la scène arrive souvent uniquement parce qu'il l'a décidé, sans qu'il se soucie de légitimer autrement leurs actions" (p. 25).

(1) Die Bedeutung der soph. Elektra fir den Aufbau der Ilundlung, diss. de Munich, 1930, p. 22.

(2) Mélanges Weil, p. 335, (p. 3 du tirage à part). 
d'Eschyle moins sévèrement que ses censeurs. Ainsi, peut-on dire que le passage d'Agamemnon où le coryphée s'enquiert de Ménélas (617 sqq) est inutile à l'action? Ménélas est l'héritier d'Agamemnon pendant la minorité d'Oreste, et tous les spectateurs ont présents à l'esprit les deux passages d'Homère où il est désigné comme son vengeur naturel $\left(^{1}\right)$. Il est donc du plus haut intérêt pour tout le monde dans le palais de savoir si Ménélas et sa flotte suivent ou non le roi d'Argos. Après la réponse du héraut, le chœur ne peut plus espérer l'intervention qui arrêterait ce qu'il redoute obscurément $\left({ }^{2}\right)$.

De même il est inexact de dire que, si Cassandre refuse de suivre Clytemnestre et si Clytemnestre n'insiste pas pour la faite entrer, c'est sans raison (la thèse de l'auteur, on s'en souvent, c'est que cette scène inutile sert simplement à contrepeser la scène utile qui lui correspond dans les Choéphores $\left({ }^{3}\right)$. Cassandre refuse d'entrer parce qu'elle a peur; lorsqu'elle se ravise et entre enfin, c'est de sa part une véritable résolution de suicide, qui est très clairement indiquée dans le texte ;

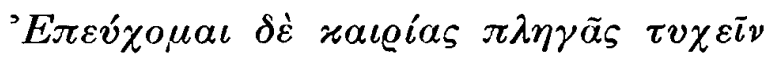

et :

$$
\text { 'A }
$$

Clytemnestre n'ose pas la contraindre de peur de donner l'alarme à Agamemnon. Voir une contradiction entre le refus d'entrer, puis l'entrée volontaire au vers 1362 , c'est méconnaître tout le génie psychologique d'Eschyle.

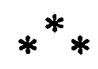

(1) Odyssée, IV, 546 et III, 255.

(2) Parmentier lui -même, fort indifférent par ailleurs à ce qu'on appelle la technique dramatique, estime invraisemblable qu'Oreste laisse sans nécessité ignorer à sa sceur qu'il est vivant. Il n'y a nulle invraisemblance : le désespoir d'Electre est nécessaire pour rassurer Clytemnestre. $A$ vrai dire, auprès d'Egisthe, Électre saura feindre. Mais un conspirateur sait fort bien qu'un complice, surtout aussi passionné, ne peut feindre longtemps.

(3) p. 18. La note 14, p. 229, est au surplus inexacte. C'est Eschyle et non Euripide qui les premier a fait Clytemnestre jalouse d'Agamemnon. Il est difficile de savoir si Cassandre aime Agamemnon ,mais, ce que est sùr, c'est que Clytemnestre en est persuadée, le crie bien haut et s'en tient outragée : $\varkappa \varepsilon i \tau a \iota$

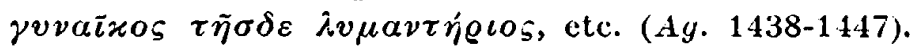


Celui qui recherche les détails rudimentaires à l'intérieur des tragédies est tenté de substituer à l'étude des poètes l'étude des thèmes, qui coupe la première à angle droit. Et cela ne va pas sans inconvénients. Par exemple, M.Stoessl estime que le personnage de Chrysothémis a été inventé par Sophocle ( $\left.{ }^{1}\right)$ mais que celui d'Ismène appartenait à une tradition antérieure, du reste inconnue, où Antigone jouait un ròle accessoire $\left({ }^{2}\right)$. Or, le goùt de Sophocle pourles oppositions de caractères du type Antigone-Ismène, ÉlectreChrysothémis, avait déjà frappé less Anciens $\left({ }^{3}\right)$. Que, dans les sept pieces conservées, on trouve deux couples de ce genre, cela indique une prédilection du poète pour les contrastes psychologiques.

M. Stoessl objectera que, l'apport personnel de Sophocle, c'est l'individualisation du personnage, et que ce travail de création s'est fait, que la Vorlage apportât simplement un nom - Chrysothémis - , ou qu'elle apportât un thème tout élaboré comme il le suppose pour le cas d'Ismène. Cela est exact, mais l'erreur reste tout de mème de traiter la Vorlage du poète comme une source d'historien et d'admettre qu'un thème donné sera toujours traité à l'intérieur de la légende mème où il est apparu primitivement. Or, cela est faux.

Ainsi, nous avons une réplique de l'arrivée de Cassandre dans Agamemnon: c'est l'entrée d'Iole dans les Trachiniennes, une captive muette, favorite malgré elle, fatale sans l'avoir voulu à la maison oì elle va entrer. I a parenté est llagrante, mais, pour la constater, il a fallu sortir du cycle des Atrides. De mème, un

(1) Démonstration pp. 58-60. On sera aisément d'accord avec M. Stoessl sur la conclusion, mais non sur ceci : "Soph. brauchle die Figur der Chrysothemis um in der Behandlung des Stoffes überhaupt seine eigene Wege gehen zu können.

(2) Démonstration p. 187. Cela l'amène à considérer comme pré-sophocléen le thème développé dans la dernière partie des Sept. En admettant même que ce fut démontrí, il ne s'ensuivrait nullement, comme M. Stoessl parait l'affirmer, que la fin des Sept fut authentique. Présophocléen et eschyléen ne sont tout de mème pas des termes synonymes. Zielinski, Trayodumena, p. 17, admet aussi l'authenticité de ces pages contestées.

(3) Dioscoricle. Anth. Pal, VII, 37 : le schol. à Soph. El. 328 dit très justement que Sophocle a opposé les deux snurs l'une à l'autre pour $\delta \iota x \pi o \iota x i \lambda \lambda \varepsilon \iota v$ raís $\dot{a} \nu \tau \iota \varrho \varrho \eta ́ \sigma \varepsilon \sigma \iota \tau \dot{a} \delta \varrho \alpha ́ \mu \alpha \tau \alpha$. Au surplus, si, dans la poésie. Chrysothémis n'apparail avant Sophocle que dans l'lliade (IX, 287), un peintre attique l'a représentéc assistant Oreste (Robert, Bild und Lied, p. 155). Qui ne voit combien il serait facile, en utilisant cette version de la légende. de démontrer que Chrysothemis vient d'une mythopée antérieure? 
poète s'est inspiré du ressort principal de Philoctète : c'est Virgile dans l'épisode de Sinon. Dans les deux cas, une étude limitée à une seule légende ne nous apprend rien. Pour les tragiques grecs, un thème mythique est surtout le support d'une construction psychologique. On peut faire des conjectures assez plausibles sur la structure des histoires qui servent de cadre aux tragédies perdues, mais la plausibilité décroît à mesure qu'un détail apparaît davantage comme chargé de psychologie, parce que, dans ce cas, le génie créateur joue à plein et remanie la Vorlage qui est peut-être, au surplus, tout autre chose que ce que nous croyons.

Il faut, en effet, une certaine naïveté pour s'imaginer qu'on pourra reconstruire le Lä̈os d'Eschyle en retirant, d'EEdipe-Roi, tout ce qui semble être l'apport personnel de Sophocle (et,ô merveille, il ne reste en effet que des scènes à deux acteurs) ; - qu'on retrouvera l'GEdipe d'Eschyle en appliquant le même traitement à CEdipe à Colone (pp. 196-255); - que Sophocle. pour écrire Electre, a longuement médité sur les Chéophores, étudiant l'économie de la pièce et remplaçant délibérément tel personnage par tel autre. Il a réfléchi au problème posé par Eschyle, ce qui n'est pas la même chose, et, là où nous croyons saisir une influence, c'est peut-être celle, non d'Eschyle, mais de quelque œuvre perdue qui était peutètre médiocre. En tout cas, plutôt que de comparer les deux œuvres scène à scène depuis le premier vers $\left({ }^{1}\right)$, mieux vaudrait encore prendre les deux pièces par le dénouement et constater que, pour Eschyle, il y un problème religieux du matricide, de telle sorte que les faits se présentent en gradation ascendante: meurtre d'Egisthe, meurtre de Clytemnestre, trouble et doute, apparition de Furies. Dans son Electre, Sophocle, comme Homère, voit surtout dans la succession d'Agamemnon un problème dynastique, si bien que les faits s'ordonnent tout autrement: meurtre de la mère, meurtre de l'usurpateur, acte plus dangeureux et plus décisif que le premier, triomphe de la «branche aînée " rétablie dans ses droits. Que les Furies n'apparaissent pas à la fin d'Electre, M. Stoessl le signale à peine (p. 82, à propos d'Euripide) tant, en philologie, il est plus malaisé de distinguer les absences que les présences.

(1) La symétrie que M. Stoessl découvre (p. 63), entre les Choéphores et Electre, dans l'allusion à Io qui figure au $5^{\mathrm{e}}$ ou au $6^{\mathrm{e}}$ vers des deux pièces, repose sur une méprise, puisque le prologue des Choéphores est mutilé et restitué par conjecture. 
Et, cependant, cette privation domine toute la pièce. I a conception générale que Sophocle se fait du monde, du devoir, des dieux, modifie ici l'économie même de la tragédie. Mais les modifications ne se marquent pas au début de l'œuvre. Et l'on voit alors avec quel grain de sel il convient de prendre ce que M. Stoessl dit du prologue. Dire que, dans le prologue, l'auteur annonce ses propres innovations et souligne le point où il se détache de la mythopée antérieure, c'est énoncer un fait qui est presque toujours exact et qui, du reste, s'explique fort bien : le poète empèche le spectateur de s'engager sur la fausse piste de ses souvenirs et lui donne tout de suite le bon aiguillage ( ${ }^{1}$ ). Mais on n'a pas le droit d'en conclure que cela soit une règle générale et que tout prologue donne la clef de toutes les innovations d'un drame (pp. 65, 79, 190). La composition d'une ceuvre d'art ne se ramène pas à la solution d'un problème de technique.

La détection des détails rudimentaires aide à deviner la mythopée antérieure. Mais il faudrait reconnaître que les poètes ne subissent: pas passivement les formes légendaires, comme la baleine porte, sans le savoir, son fémur minuscule. Si Euripide, après avoir imaginé qu'Oreste tue Pyrrhus, envoie néanmoins celui-ci mourir à Delphes, c'est que cet épisode lui convenait, pour des raisons que nous n'avons pas à relever ici. De mème, il est certain que l'épisode de la tresse d'Oreste sert à la reconnaissance du frère et de la socur, chez Eschyle mais non chez Sophocle, où la preuve est faite grâce à l'anneau d'Agamemnon, ni chez Euripide, où le vieux pédagogue identifie directement le fils du roi mort. L'offrande des cheveux, dans les deux dernières pièces, est cependant tout autre chose qu'un détail inutile: Electre en est troublée, agitée de sentiments contraires, préparée à des émotions violentes. Pour un poète tragique, un épisode peut servir à autre chose qu'à laire avancer l'action. M. Stoessl n'y voit pas grand chose de plus qu'un rouage dans le mécanisme scénique.

(1) M. Stoessl met l'accent sur le fait que. dans le prologue, le poète s'oppose a ses devanciers. Il y a donc, au début d'une tragédie, une sorte d'intention critique, et "les prologues de Térence apparaissent comme des descendants lointains, mais directs, des prologues tragiques" (p. 79). Idée curicuse, (1u'on devrait creuser. 
Et cela lui dicte parfois des appréciations étranges. Par exemple, dans l'Electre d'Euripide, il constate que le Laboureur et le Pédagogue sont de simples "utilités" qui, du reste, se doublent. L'un d'eux doit venir de la mythopée antérieure : ce sera le Pédagogue, qui ressemble à celui de Sophocle; l'autre doit être euripidéen : ce sera le Laboureur, qui fait le prologue, lieu où le poète annonce communément ses innovations (p. 77). Au surplus. "le rôle dramatique du Laboureur se borne à aller chercher le Pédagogue " ( $p$. 69). Ceci appelle deux remarques. D'abord, tout lecteur candide rapprochera pour ce point Electre, non de la pièce de Sophocle, mais d'une pièce contemporaine d'Euripide lui-même, Ion, où apparaît également, à côté d'une princesse dans la peine, un vieil esclave secourable. Euripide a dù aimer ce contraste, comme Sophocle a aimé le contraste de la sour énergique et de la sceur timide. Le Pédagogue apparaît donc, non comme un "personnage rudimentaire ", (p. 96), mais comme une création foncièrement euripidéenne. Wilamowitz dirait peut-être, comme il le disait de Sophocle faisant de Chrysothémis une réplique à Ismène, qu'« Euripide s'est copié lui-mème ». Entendons par là que, si une légende fait une ensemble, l'œu vre d'un poète est un autre ensernble, à tout prendre plus solide et plus cohérent. Car il est impossible d'étudier la légende en soi comme une réalité indépendante. Elle n'existe et ne se comprend pas en dehors du poète.

Quant au Laboureur, il est tout autre chose qu'un ressort dramatique. Sa présence met Electre dans cette atmosphère de déchéance qui renouvelle si curieusement tout le sujet. Mais les critiques allemands ne voient de renouvellement possible que par la technique pure, c'est à dire dans la solution d'un problème à deux exigences, la première étant la vraisemblance et la seconde l'émotion. Or, ici, nous sommes de mauvais juges. Un lecteur peut avoir l'impression que l'événement qu'il lit manque de la $\pi \iota 0 \alpha \nu o ́ \tau \eta \varsigma$ qu'on exige dans la vie courante; mais le spectateur, plus ému que nous, a pu avoir une impression toute différente. Et dire que le poète a sciemment sacrifié la vraisemblance à l'effet, c'est nc voir cn lui qu'un artisan, dans l'atelier duquel on prétend s'introduire en croyant qu'on va le surprendre au travail. Cette tendance moderne de l'exégèse allemande a été finement critiquée par Lesky (1),

(1) Die Orestie des Aeschylos, Hermes, 1931, pp. 190 sqq. Je renonce à tirer quoi que ce soit de l'étude de Snell, Aischylos und des Handeln im Drama (Philo- 
lequel marque très bien ce qu'a de dangereux la prétention du handuerksmässig à la primauté et il dit: "On procède trop souvent comme si une analyse technique pouvait dégager un squelette, la composition à l'état pur, discernable indépendamment du contenu spirituel de l'ouvre d'art». M. Stoessl reconnaît du reste très honnètement au seuil de son enquète, qu'il n'étudiera, chez Eschyle, que le travail conscient de l'artisan et que c'est là l'aspect le plus humble de la création poétique. Pourquoi faut-il qu'après de si sages prémisses, l'auteur aille reconstituer des pièces entières d'après une loi inférée d'un seul exemple, exactement comme si la composition existait en dehors des choses composées?

Peu avant le livre de M. Stoessl avait paru celui de M. Méautis, consacré également à la théologie eschyléenne. ( $\left.{ }^{1}\right)$ Trilogie et non tétralogie. Seuls, ceux qui étudient l'histoire des concours tragiques s'attaquent au difficile problème des ensembles quaternaires. La plupart des critiques considèrent la trilogie comme un tout. Ainsi faisaient déjà, très probablement, Aristarque et Apollonius, qui parlaient la trilogie (Orestie, abstraction faite du drame satyrique $\left({ }^{2}\right)$.

Au surplus, il est inutile de rappeler ici à quelles difficultés l'on se heurte lorsqu'on essaie de savoir quelle "unité " un poète présentait à un concours. Les noms mèmes de tétralogie et de trilogie ne semblent pas ètre antérieurs à l'époque alexandrine $\left({ }^{3}\right)$. Christ, étonné qu'on appelât une tragédie un $\lambda o ́ \gamma o s$, a émis l'idée que le nom de tétralogie, loin d'avoir passé de la tragédie au dialogue philosophique, pourrait bien avoir suivi le chemin opposé, ce qui lui enlève toute signification pour une époque ancien-

logus, Suppl. XX, 1928, pp.1-16.4). Il est peu probable en effel qu'on puisse approcher du problème constituc par la tragedie en essayant de préciser les no-

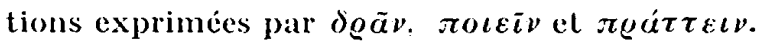

(1) Eschyle et la trilogie, l'aris, cirasset, 1936.

(2) Schol. d'Ar. Giren. 1124... Isener (Inser Platontext, Nachr. der K. Ges. der Wiss. zu (:öll., 1892, pp. $213 \mathrm{sqq}$ ) en concluail, peut-ètre sans raison suffisante, que ces critiques étudiaient systématiquement la trilogie, indépendamment des jeux satyriques. - Welcker aussi itudie die Aeschyl. Trilogie. IIermann au contraire a ecrit sur la Tetralogia.

(3) Avant cela, on dit $\delta \iota \delta \alpha \sigma \varkappa \alpha \lambda i \alpha, \chi o \varrho o ́ s, \tau \varrho \alpha \gamma \omega \delta i \alpha$. On trouvera tous les textes réunis et critiqués dans l'étude de Wiesmann analysée ci-dessous. 
ne (1). F. Schoell $\left({ }^{2}\right)$ a essayé de démontrer naguère que les chiffres transmis pouvaient, pour chaque auteur, se diviser par quatre et que la structure tétralogique était la seule normale. Mais la didascalie de 467 dit qu'Aristias concourut avec trois pièces seulement contre Eschyle et Polyphradmon qui en présentaient quatre. Et la notice de Suidas, du reste altérée et douteuse, qui dit que Sophocle le premier concourut avec des pièces détachées, comment la concilier avec le fait que les Perses n'appartiennent pas à une tétralogie liée? Il paraît impossible de saisir où que ce soit, ou une coutume fixe, ou un principe d'évolution.

Maurice Croiset, il y a un demi-siècle, a essayé de le faire $\left(^{3}\right)$. Il part d'un passage de la Poétique (c. 5) où Aristote dit que la tra-

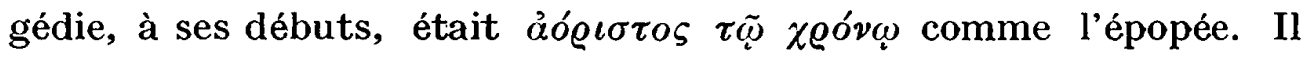
en infère l'existence d'une tragédie longue et diffuse où était intéressé le chour tout entier, avec ses cinquante choreutes. Un groupe de choreutes fut d'abord séparé des autres et affecté au drame satyrique, puis les rôles se distribuèrent entre les autres fractions du choeur et, ainsi, "des tragédies partielles se formèrent spontanément dans la tragédie totale ". Le remplacement d'un chœur partiel par un autre permettait de diviser d'une manière simple et agréable une matière trop longue" (p. 375). Mais pourquoi ce sectionnement en trois parties, et non en deux ou quatre? "Cette division ternaire répondait mieux que toute autre à la succession naturelle des choses, puisqu'elle se prêtait à marquer dans une série d'événements le commencement, le milieu et la fin (p.376). Ainsi, l'histoire de la forme tragique apparaît conforme à "l'ordre naturel des choses qui veut que les formes se succèdent dans l'art comme dans la vie en suivant une loi d'évolution" (p. 379). L'apparition de la tétralogie s'est faite "par un simple phénomène de végétation naturelle, par une sorte de sectionnement organique résultant de la vie même" (p. 374). Je cite ces phrases : elles prouvent que si, en 1937, on est un peu trop incliné à voir dans le poète un technicien de la matière légendaire, en 1888 on prenait les formes d'art pour le résultat d'une floraison spontanée, mûrissant toutes seules

(1) Christ, Platonische Studien, Abh. Bayer. Ak. 1885, pp. 460 sqq. - cf. Hirzel, der Dialog, I, 1895, pp. 253 sqq.

(2) F. Schoell, Ueber zwei Trilogien des Eurip. Abh. Heidelb. Ak. 1910 fasc. 15.

(3) De la tétralogie dans la trag. grecque, Rev. Et. gr., 1888, pp. 369 sqq. 
comme des pommes sur une branche. $\Lambda$ vrai dire, les chercheurs des dernières années, qui demandent tout aux textes et rien aux lois mystéricuses de l'évolution naturelle et du transformisme littéraire, n'arrivent pas à un résultat plus positif,

M. P. Wiesmann ( ${ }^{1}$ ) part essentiellement des didascalies, seuls documents contemporains et sûrs. Et il aboutit à la conclusion la plus négative : la composition tétralogique vient de conditions purement extérieures, notamment du fait que trois pauses coupaient bien la journée en quatre parties et donnaient des auditions proportionnées à la résistance des auditeurs. La composition tétralogique n'a donc rien à voir avec la nature même de l'art tragique $\left({ }^{2}\right)$; on pouvait l'abandonner et, dans certains cas, ne donner que trois ou deux pièces. La cohésion interne ne fut à aucune époque une nécessité. M. Wiesmann entreprend même de démontrer qu'elle n'a jamais existé et que les trois pièces de l'Orestie sont liées par un lien faible et lâche, chacune d'elle, en revanche, étant fortement nouée (auf sich selbst konzentriert) $\left(^{3}\right)$. Le poète a rattaché l'une à l'autre lés pièces qu'on devait jouer le même jour, et l'unité, loin d'être primaire, résulte d'une pure contingence. Le jeu satyrique, certainement joué en dernier lieu, se place chronologiquement entre Agammenon et les Choéphores; même une trilogie liée n'offrait done pas le développement régulier d'une action unique.

La partie faible de cette argumentation saute aux yeux : s'il n'y a eu primitivement aucun lien nécessaire entre les tragédies jouées le même jour, si la composition par quatre n'est ni primitive ni obligatoire, comment s'expliquer qu'elle se soit généralisée malgré toutes les gênes qu'elle imposait aux poètes, aux chorèges, aux juges et même au public? Et ces circonstances extérieures et contingentes qui l'ont généralisée, quelles sont-elles? Pourquoi trois pauses? A quels moments de la journée? On est tout disposé à renoncer avec M. Wiesmann à l'idée d'un «développement or-

(1) Das Problem der tragischen Tetralogie, diss. de Zürich, 1929.

(2) Ni M. Cantarella (I primordii della tragedia,Salerne, 1936), ni M. Pohlenz (Die griech. Tragödie, Teubner, 1930) n'étudient le problème de la tétralogie. Si bizarre que cela puisse paraitre, lorsqu'on scrute les origines de la tragédie, on ne rencontre la tétralogie nulle part. C'est véritablement une énigme adventice.

(3) Comme MM. Stoessi et Lesky, M. Wiesmann estime qu'Ag. et Choéph. se ressemblent étroitement par la conduite de l'action Cf. supra p. 35. 
ganique "qui aurait transformé l'Urtragödie en tétralogie liée et celle-ci en tétralogie libre, métaphore biologique qui autorise bien des à-peu-près. Mais, dans l'hypothèse que M. Wiesmann présente sans conviction, on ne voit rien qui commence seulement à expliquer les faits.

\section{$* * *$}

Les critiques allemands dont nous avons parlé partent de la notion aristotélicienne de l'action. M. Méautis refuse à cette poétique toute valeur pour l'interprétation des tragédies. Tout ce que nous pouvons savoir sur l'esthétique tragique se trouve dans les œuvres elles-mêmes. Voilà un bon point de départ : lire le texte et chercher bonnement à le comprendre .M. Méautis entreprend bientôt, du reste, d'aller plus loin. Alors que M. Wiesmann voit dans le groupement trilogique un phénomène contingent et sans importance, M. Méautis y découvre le principe mème de l'art d'Eschyle, lequel a probablement imaginé de s'imposer à lui-même cette féconde contrainte (pp. 44-45). Il n'est nullement impossible qu'Eschyle ait en effet inventé la trilogie, mais à vrai dire nous n'en savons rien. Il est vrai aussi que "pour un homme comme Eschyle le problème de la vie ne s'arrête pas au problème de l'individu; il doit être étudié au fonction de la famille et de la race qui, seules, permettent de le comprendre ". Mais, après tout, cet intérêt pour les aspects successifs d'une question aurait pu s'exprimer autrement que par la succession trilogique. Et, à vrai dire, M. Méautis, non seulement ne nous dit nullement comment il conçoit la trilogie eschyléenne, mais il se borne, à propos des pièces perdues, à quelques mots empruntés aux préfaces de M. Mazon. On trouvera dans son livre de bonnes analyses des pièces conservées, mais rien de précis sur le problème de la trilogie.

Celui-ci devrait ètre posé sur le plan de la religion et de la morale, comme $M$. Méautis le souhaite, mais avec plus de précision et de clarté que lui-même ne l'a fait. Il semble, en effet, que la structure tétralogique a permis à Eschyle, tantöt de traiter successivement les différentes phases d'une légende (CEdipodie), tantôt de montrer successivement l'âme de vérité de chacune des thèses en présence (Suppl., Prom.), tantôt enfin de superposer, à l'étude d'un caractère, l'étude d'un problème (Orestie). M. Méautis se pose la question de savoir si le personnage de Clytemnestre évolue entre Agamem- 
non et les Choéphores. Je crois qu'il a raison de répondre négativement. Ce qui a changé, c'est le point de vue d'Eschyle. Dans Agamemnon, il montre une femme de chair et de sang qui a ses raisons et ses torts; à partir des Choéphores et surtout dans les Euménides, il ne s'agit plus que d'un problème de droit familial considéré presque abstraitement. Les actes comptent seuls; les mobiles individuels s'effacent, parce que le poète veut arriver à une solution universellement valable. Agamemnon est un tableau, les Euménides sont une épure. Il ne paraît pas du tout qu'il y ait un glissement analogue dans les trois ensembles auxquels appartiennent les Sept, Prométhée, les Suppliantes. Le peu que nous savons des deux trilogies qui comprenaient, l'une les Troyennes, l'autre les Phéniciennes, semble indiquer qu'Euripide, comme Eschyle dans l'CEdipodie, aimait à dérouler dans la durée des enchaînements de faute et de sanction. Peut-être serait-il malaisé, vu le petit nombre des documents, de dépasser ces quelques indications sans recourir à d'hypothétiques reconstructions qu'il vaut mieux éviter.

Et on pourrait aussi s'attacher à des questions mineures. L'étrange Oreste d'Euripide ne pouvait guère tenir lieu d'un drame satyrique, mais ne serait-il pas l'équivalent d'une troisième tragédie et du drame satyrique? N'y aurait-il pas eu des cas où un ensemble tenait lieu de deux éléments tétralogiques? Cela pourrait expliquer des chiffres dont nous n'arrivons pas à rendre compte aujourd'hui. 